\title{
NOTAS A LOS REGISTROS NOTARIALES DE JUAN CAMPOS
}

\author{
M. ${ }^{a}$ Luisa Cabanes Catalá \\ Universidad de Alicante
}

La documentación e institución han sido objeto de abundantes estudios, en cuya enumeración no voy a entrar, si bien hay algún aspecto, tal es el de los registros que quizá ha sido algo olvidado.

En los notarios valencianos se ha constatado el hecho, ya señalado por Boüard (1), que un notario podía llevar hasta tres libros registros sincrónicos en el tiempo, si bien en su contenido presentan algunas diferencias. Estos volúmenes aparecen designados bajo las denominaciones de reebedor, protocolo y notal. La existencia de éstos, en el Reino de Valencia, fue detectada primero por Dualde (2) cuando realizó el inventario de parte de los fondos notariales del Archivo Municipal de Valencia. A juicio de este autor el primero de los volúmenes, el reebedor, sería el que contendría los datos indispensables para la posterior redacción del documento. En el protocolo se registrarian los instrumentos ya redactados, si bien se habría procedido a la sustitución por etcétera de las fórmulas. Finalmente, el notal recogía el documento redactado ein extenso", señalando Dualde que en muchos casos los textos están sólo iniciados.

Posteriormente Rodríguez Troncoso (3) detecta la presencia de estos tres registros notariales en el Archivo del Reino, y será precisamente sobre los de un notario de este archivo del que hemos hecho el estudio.

Por su parte, Ferrer i Mallol, en su estudio sobre la elaboración del documento notarial (4), menciona a finales del siglo XV o en los inicios del $X V I$ que los dos registros iniciales que portaban los notarios catalanes se transforman en tres: «Es converteix en un sistema de triple registre en apèreixer el manual petit, generalment en octau, pero també a vegades en dotzan, dit manualetum, prothocollum, manuale sive repertorium o re- 
pertorium, a vegades vademecum i també manuale aprisiarium o simplement aprisie, noms que foren subtituis en el $\mathrm{s}$. XVII per més general de borrador" (5). Según esta autora, el libro pequeño servía para tomar los datos delante del cliente y su escritura era cursiva, con numerosas correcciones, con posterioridad el documento se pasaba al «manual», que tendría una presentación más cuidada y en tamaño folio, el último paso sería su traslado al "liber contractium comunium". Sin embargo, a su juicio, cada notario llevaba su propio sistema (6).

La legislación foral recoge diferentes menciones a estos libros. La primera referencia aparece en los fueros de Jaime I, en la rúbrica XIX y regula la necesidad de que cuando el notario sale de la ciudad, sus libros deberán quedar en manos de otro notario.

"Los scrivans quam se morran o partiran de la ciutat esniran en altre loch per rao dabitar o de estar iaques quen lo libre el memorial e les cartes que seran closes a alcun notari habitador de la ciutat: lo qual notari en loch daquell qui sera absent pusque fer les cartes e metre les notes en forma publica" (7).

Puede verse que en esta primera mención a los registros notariales no hay ninguna concreción a los mismos.

En esa misma rúbrica vuelve a mencionar los libros, y, en esta ocasión, es algo más concreto: «...e ells ab lurs propries mans scriven e facen notes el libre o el memorial de les notes" (8). De este texto parece desprenderse la posibilidad de la existencia de dos libros diferentes.

Nuevamente, en los fueros de Pedro IV se les menciona con motivo de prohibir a las personas privadas que los tengan: «E semblantment alcunes vegades les dites privades persones tinents los dist libres o protocols o scriptures en frau de aquelles de qui sera interes..." (9).

La última mención que encontramos corresponde a Juan II, cuando es lugarteniente de su hermano, "ordenam que los prothocols o libres notals e judicials mort lo notari no puxen restar sino en poder de notari..." (10).

A través de estos textos puede verse que apenas hay una concreción sobre los diferentes "tipos" de registros que se han conservado de algunos notarios. Por su parte, los documentos de nombramientos de notarios no son tampoco muy explícitos, tomemos como ejemplo la referencia que en dispositivo del documento de nombramiento como notario de Pedro Galbe de Orihuela, quien podrá ejercer como notario en el Reino de Valencia hasta Jijona, "...de quibus quidem [se refiere a los diferentes documentos que puede extender] prothocolla sive capibrevia faciatis notularum ut eterne memorie comendentur" (11).

Una de las dificultades que se plantea el estudio de estos tres volúmenes está motivada por la falta de conservación del primero de ellos, el reebedor, desconociendo si por la misma característica del volumen, 
como veremos luego, no los guardaba el notario porque tomaba esos primeros datos en una hoja de papel que al pasar al protocolo destruía.

Hemos tomado los volúmenes conservados del notario Juan Campos (12) correspondientes a 1477, 1478 y 1479. El análisis que de ellos hemos realizado se ha basado en dos puntos:

- autodenominación de los volúmenes y caracterisitcas de los mismos;

- análisis estadístico de uno de los años comprendidos en ellos, siendo elegido 1478.

Respecto al primer punto, y en lo referente al reebedor, al comenzar los documentos correspondientes al año 1478 leemos:

"Prothocollum mei lohannis de Campos, auctoritate regia notarii publici Valencie ac per totam terram et dominacionem serenissimo domini regis Aragonum confectum sub anno $\mathbf{M}^{\circ}$

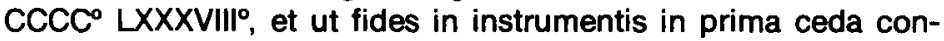
tentis, per me receptis et propria manu mea scriptis atribuatur meum artis notarie assuetum, appono in testimonium premissorum, si g[signo]num» (13).

El notario inicia con la genérica denominación «prothocollum», para luego especificar que es la de primera redacción.

Es un volumen en cuarto, que en ocasiones presenta saltos cronológicos. Su aspecto exterior es desordenado y da la impresión que son un conjunto de notas tomadas en hojas sueltas y que posteriormente se han encuadernado.

El segundo de los volúmenes, el protocolo, comienza los documentos correspondientes al año objeto de nuestro estudio con el siguiente texto:

«Prothocollum mei lohannis de Campo auctoritate regia notarii publici Valencie ac per totam terram et dominacionem serenissimi domini regis Aragonum, confectum sub anno a Nativitate Domini $\mathrm{M}^{\mathrm{mo}}$ sepuagesimo octavo, et ut fides in instrumentis in presenti prothocollo contestis et manu propria scriptis adhibeatur meum artis notarie assuetum, appono infidem et testimonium premissorum sig [signo] num» (14).

Este segundo libro, que aparece denominado como "protocolo" en la propia documentación, es un volumen de tamaño cuarto, pero de aspecto más ordenado y de una mayor claridad de escritura, dentro naturalmente de una escritura sursiva. El instrumento aparece redactado en forma, aunque el desarrollo de las fórmulas aparece algunas veces sustituidas por etcétera, según Dualde (15); sín embargo, en el volumen por nosotros manejado este hecho no es constante, ya que en ocasiones el documento está redactado con todas sus fórmulas desarrolladas, e incluso no faltan 
los casos de la doble forma, es decir, que podemos encontrar un documento con las fórmulas acortadas por el etcétera y a continuación el mismo documento con las fórmulas desarrolladas por completo.

Queda finalmente la mención a cómo se inicia cada año en el denominado «notal»:

"Liber notularum mei lohannis de Campos, auctoritate regia notarii publici Valencie ac per totam terram el dominacionem serenissimi domini regis Aragonum, confectus sub anno a Nativitate Domini $\mathrm{M}^{\mathrm{mo}} \mathrm{CCC}^{\circ}$ septuagesimo octavo, et ut in eo fides in dubia atribuatur meum artis notarie assuetum in presenti apono sig [signo] num» (16).

De la comparación con los preámbulos anteriores destaca de ellos porque no ha usado la denominación genérica "prothocollum", sino que ha matizado su nombre "liber noturalum». Hay además otras diferencias de carácter formal. Es éste un volumen en tamaño folio, a diferencia de los dos anteriores que son en cuarto, de buena caligrafía y que destaca especialmente por la gran cantidad de hojas que aparecen blancas o que contienen solamente el inicio de los documentos.

Pero, junto a estas diferencias que afectan a la autodenominación y características de los volúmenes, hay otras que afectan a los documentos allí contenidos y que ofrecemos en el siguiente cuadro, indicando que el estudio y análisis se ha realizado en los documentos correspondientes a 1478.

\begin{tabular}{lcccccc}
$\begin{array}{c}\text { Tipo } \\
\text { registro }\end{array}$ & $\begin{array}{c}\text { N. Do- } \\
\text { cumentos }\end{array}$ & Latín & $\begin{array}{c}\text { Valen- } \\
\text { ciano }\end{array}$ & $\begin{array}{c}\text { Latín- } \\
\text { Valenciano }\end{array}$ & $\begin{array}{l}\text { Cance- } \\
\text { lados }\end{array}$ & $\begin{array}{c}\text { No can- } \\
\text { celados }\end{array}$ \\
\hline $\begin{array}{l}\text { Reebedor } \\
\text { Protocolo }\end{array}$ & 59 & 20 & 38 & 1 & 54 & 5 \\
Notal & 54 & 45 & 9 & - & 9 & 45 \\
\hline
\end{tabular}

En el "reebedor» hay anotados 59 documentos correspondientes al año 1478 , de ellos, como puede verse, la mayoría aparecen escritos en valenciano, en total 38, mientras que en latín lo están sólo 20. Dándose además la circunstancia que uno de ellos lo está en ambas lenguas. Los documentos que han sido cancelados, y por tanto se pasaron al segundo registro, fueron 54, figurando además en el “reebedor» una sentencia arbitral, pero el número se mantiene igual a los cancelados en éste, porque de aquéllos uno no se copió en el protocolo.

En el segundo volumen, el protocolo, el número de documentos que 
encontramos es de 54 , de los cuales 45 han sido redactados en latín y sólo 9 en valenciano; esto no es de extrañar porque la redacción del documento se hacía usualmente en latín, si bien el notario a petición de parte podía expedirlo en valenciano (17). Por tanto, pienso que el predominio de los documentos redactados en valenciano en el "reebedor" debía obedecer a que las notas se tomaban en el lenguaje habitual. Más sorprendente si cabe es el número de documentos cancelados, sólo 9 , mientras que 45 no lo están. Siguiendo el razonamiento utilizado para el primer registro, aquéllos serían los que deberian pasarse al "notal». Sin embargo, en el mencionado libro y correspondiente al año 1478 no hay ningún documento pasado por completo, pero están iniciados todos los documentos que aparecen en el «protocolo» menos 2, pero, además, están asimismo comenzados 3 que no figuraban en el «protocolo», pero sí en el «reebedor».

Todo lo expuesto, somos conscientes de que son unas breves notas sobre los diferentes volúmenes que podían llevar los notarios y que habrá que concretar más con estudios realizados sobre los registros notariales de otros notarios, para ver si esas pinceladas que se perfilan pueden concretizarse en unas características comunes a todos ellos.

Como apéndice documental incluimos un documento de 1479 en sus tres fases (18).

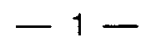

1479, marzo, 12. Valencia

Protocolo. Apoca firmada entre Marcos de Castrellenes, labrador de Mislata y Leonarda, viuda de Jaime de Castrellenes.

\section{A.R.V.Protocolos, 440}

Die veneris intitulata XII marcii anno $\mathrm{M}^{\circ} \mathrm{CCCC}{ }^{\circ} \mathrm{LXVIII)^{ \circ }}$.

Marcus de Castrellenes, laborator loci de Mizlata firmavit apoquam domne Leonarde, uxoris quondam lacobi de Castrelles, laboratoris orte Valencie, de XIII libris, VI solidis, III denariis quos ego ipse Marchus bistraxit in uno pano sive bruneta dehuytena empta ad oppus sepulture dicti lacobi de Castrellenes et ex alia parte LX solidos quos ipse graciosse mutuavit dicto lacobo, et cetera.

\section{Actum Valencie.}

Testes Miquael Garcia, notarius et lohannes Domingo, virgarius baiulie generalis. 
1479, marzo, 12. [Valencia]

Reebedor. Apoca firmada entre Marcos de Castrellenes, labrador de Mislata y Leonarda, viuda de Jaume de Castrellenes.

\section{A.R.V. Protocolos, 441}

Die veneris XII marcii, anno $\mathrm{M}^{\circ} \mathrm{CCCC}^{\circ} \mathrm{LXXVIIII^{ \circ }}$.

Marcus de Castrellenes, laborator loci de Mizlata firmavit apoquam domne Leonarde de (1) ussuffructuaris dels bens d'en Jaume de Castrellenes, present e amptant als seus XIII0 Iliures, VI sous, IIII diners los quals yo bestragui en una bruneta de huytena que servi per a la sepultura del dit en Jacme de Castrellenes, e d'altra part sexata sous que li presta al dit deffunt et quen.

Testes Miquael Garcia, notari, en Johan Domingo, verger de la cort.

1479, marzo, 12. Valencia

Notal. Apoca firmada entre Marcos de Castrellenes, labrador de Mislata y Leonarda, viuda de Jaime de Castrellenes.

A.R.V. Protocolos, 3531

Die veneris intitulata $X I^{\mathrm{a}}$ marcii anno a Nativitate Domini $\mathrm{M}^{\circ} \mathrm{CCCC}^{\circ}$ LXXVIII'.

Sit (1) omnibus notum quod ego Marchus de Castrellens, laborator loci de Mizlata, scienter et gratis et in veritate recognosco vobis domne Lenardo uxoris quondam / et usufructuarie omnium bonorum, quondam fuerunt lacobi de Castrellenes, laboratoris comorantis in orta Valencia, absenti et vestris quod dedistis et solvistis michi me omnimode voluntati realiter numerando omnes illas sexdecim lliuras, sex solidos, III denarios monete regalium Valencie quos michi debetisvidelicet tres libras quas graciose mutuavi dicto lacobo de Castrellenes in cuius vita et tresdesim llibras sex solidos, tres denarios quod vobis dicte Leonarde mutuavi ad opus emendi quandam brunetam sive pannum nigrum dehuite, pro sepultura dicti lacobi de Castrellenes quaquidem quantitates sumam co- 
punt predictarum sexdecim llibrarum sex solidorum et trium denariorum dicte monete, et quia rey veritas sich se renuncio scienter omni excepcioni dicte quantitatis per vos (2) dicto nomine michi tradite et non solute et per me a vobis previo nomine non habite et non recepte ut predictum doli mali.

Quod est actum Valencie, duocesimo mensis marcii anno a Nativi Domini millessimo quadringentessimo septuagesimo nono.

Sig[signo]num mei, Marchi de Castrellenes, laboratoris loci de Mizlata, predicti qui hec laudo, concedo et firmo.

Testes huius rey sunt: Miquael Garcia et Johannes Domingo, virgarius baiulie generalis ( 3 ) cives Valencia. 


\section{NOTAS}

(1) A. de BOÜARD, Manuel de diplomatique frangaise et pontificale. II l'acte privé, Paris, 1948, pp. 206-208.

(2) M. DUALDE, Inventario de la documentación notarial del archivo municipal (siglos $X I V-X V)$ en aAnales del centro de cultura Valenciana» XIII (1952) p. 392-401.

(3) R. RODRIGUEZ TRONCOSO, Documentación notarial del archivo del Reino de Valencia en ot Homenaje a J. E. Martinez Ferrando», Madrid, 1958, p. 551-553.

(4) T. FERRER I MALLOL, "La redacció de l'instrument notarial a Catalunya", en Estudios históricos y documentos de los archivos de protocolos, IV, 1974, p. 70.

(5) Ibid., p. 71.

(6) Ibid., p. 71.

(7) FURS e ordinations fetes per los gloriosos reys de Arago als regnicoles del Regne de Valencia, Valencia, 1482; Valencia, 1982, p. 178.

(8) Ibid., p. 178.

(9) Ibid., p. 288.

(10) Ibid., p. 504-505.

(11) A.R.V., Real Cancilleria 26, fol. 129.

(12) A.R.V., Protocolos 440, 441 y 2531.

(13) A.R.V. Protocolos, 441, fol. 49.

(14) A.R.V. Protocolos. 440. fol. 59.

(15) M. DUALDE SERRANO, Inventario..., p. $395 .$, .

(16) A.R.V. Protocolos 2531, fol. 47.

(17) FVRS e..., p. 282.

(18) Cuando estamos corrigiendo las pruebas de imprenta ha hecho su aparición un interesante folleto de J. BONO, Los archivos notariales, Sevilla, 1985, que recoge los manuales notariales en las pp. 17-28. 